\title{
Cancer's got nerve: Schwann cells drive perineural invasion
}

\author{
Salma H. Azam and Chad V. Pecot ${ }^{2,3,4}$ \\ 'Curriculum in Genetics and Molecular Biology, ${ }^{2}$ Department of Medicine, ${ }^{3}$ Lineberger Comprehensive Cancer Center, and ${ }^{4}$ Division of Hematology/Oncology, University of North Carolina at Chapel Hill, \\ Chapel Hill, North Carolina, USA.
}

\begin{abstract}
The invasion of cancer cells around and into nerves is associated with increased cancer aggression and poor patient outcome. As this perineural invasion increases disease severity, a better understanding of how the process is regulated may help in the development of therapeutics to target neuronal involvement in cancer. In this issue of the $J C I$, Deborde and colleagues show that direct contact between Schwann cells and cancer cells promotes cancer cell dissociation, migration, and invasion. Moreover, their data specifically suggest NCAM1 as an important molecular mediator of this Schwann cell-directed regulation of cancer cells in perineural invasion. The results of this study provide new insight into the cellular and molecular mechanisms of perineural invasion.
\end{abstract}

\section{The nerve microenvironment} and cancer progression

While the involvement of blood and lymphatic vessels in tumor growth and progression is well established, the role of nerves in the tumor microenvironment has been largely underappreciated. Increasing evidence suggests that activation of nerve growth into tumors, termed neoneurogenesis, is another key driver of cancer progression. In addition, chronic activation of the sympathetic nervous system via increased norepinephrine levels has been observed in the tumor microenvironment $(1,2)$, and this activation has been demonstrated to promote tumor growth and progression via $\beta$-adrenergic receptor signaling on tumor cells and subsequent upregulation of tumor-promoting chemokines (3-6). However, it was the hallmark paper from Magnon et al. (7) that clearly demonstrated that neuronal involvement promotes cancer progression. Specifically, this group showed that autonomic nerve formation is important for regulating primary prostate cancer growth and metastasis in mouse models and that inhibition of stromal $\beta$-adrenergic receptor and type 1 muscarinic receptor signaling inhibits tumor development and improves survival. Similarly, Zhao et al. (8) recently demonstrated that stomach denervation in mouse models of gastric cancer reduces tumor incidence and growth while improving chemotherapy response and that muscarinic acetylcholine M3 receptor loss inhibits tumorigenesis. Together, these groundbreaking studies also revealed that high tumor neural fiber density strongly correlates with tumor stage and worse clinical outcome, further implicating neuronal involvement as a key driver in cancer.

Perineural invasion is the growth and invasion of cancer cells around and into nerves present in the tumor microenvironment (9). This is believed to promote tumor progression by providing a conduit for cancer cell spread. Patients who experience perineural invasion, which has been described in many cancer types, often have poor prognoses, higher risk of local recurrence, and often suffer from severe pain $(10,11)$. In this issue, Deborde et al. uncover a key mechanism

Related Article: p. 1538

Conflict of interest: The authors have declared that no conflict of interest exists

Reference information: J Clin Invest. 2016;126(4):1242-1244. doi:10.1172/JCI86801.

of perineural invasion that is mediated by direct contact between Schwann cells and cancer cells (12).

\section{Schwann cell-mediated perineural invasion requires contact}

Schwann cells regulate neuronal growth, survival, and repair $(13,14)$ and undergo dedifferentiation into a more migratory subtype to promote neuronal guidance through matrix remodeling, paracrine signaling, and induction of neuron axonal extensions via direct cellular contact (15-18). A bundle of Schwann cells can also invaginate between individual axons to isolate them from one another (14). In this issue, Deborde et al. demonstrate that cancer exploits some of these canonical functions of Schwann cells in neuronal repair and regulation to promote cancer cell perineural invasion (12). Their findings suggest that the dedifferentiated subtype of Schwann cells associated with nerve repair is activated in cancer, as they observed a substantial increase of the dedifferentiated Schwann cell marker GFAP in tumor nerves compared with that in normal nerves in both clinical samples and a murine model of perineural invasion. In particular, only dedifferentiated Schwann cells closely associated with cancer cells, while differentiated Schwann cells did not. While Schwann cells have previously been implicated in perineural invasion based on examination of histological sections (19), Deborde et al. functionally demonstrate a cellular and molecular mechanism for Schwann cell involvement in perineural invasion (12).

In a series of beautifully intricate timelapse and fluorescence microscopy observations, Deborde and colleagues were able to document the cellular events that mediate cancer cell-Schwann cell interaction (12). In cocultures of dorsal root ganglion explants and cancer cells, cancer cells were directly recruited to neurites, and this process was dependent on the presence of neurite-asso- 

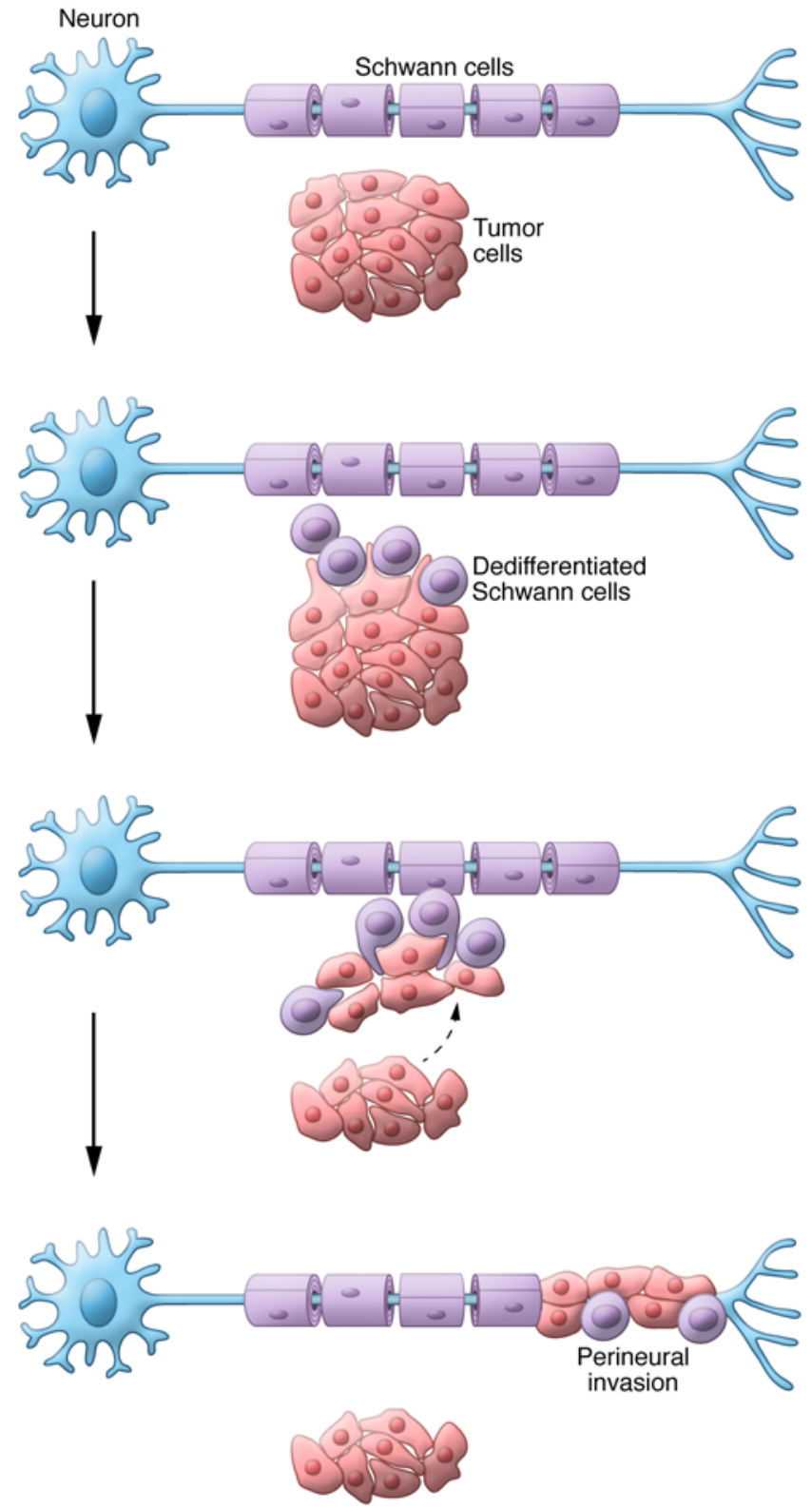

Figure 1. Schwann cells mediate perineural invasion. Dedifferentiated Schwann cells come into direct contact with cancer cells. This direct contact results in the extension of protrusions from the cancer cells. Schwann cells intercalate between cancer cells, thereby promoting cancer dispersal from the tumor and migration toward the neuron. These steps ultimately lead to perineural invasion.

ciated Schwann cells. Furthermore, direct contact between cancer cells and neurites was needed to dramatically promote migration. The authors discovered that these interactions were mediated by heterocellular contacts between Schwann cells and cancer cells. Further characterization of the precise sequence of cellular events that governs this interaction in a 3D coculture system of pancreatic cancer cell clusters and Schwann cells revealed that Schwann cells are extremely dynamic and make repetitive contacts with individual cancer cells. cell clusters to reorganize into more linear structures. At specific cell-cell sites of contact, cancer cells developed protrusions that directed their migration away from the cancer cell colony and toward Schwann cells. Interestingly, Schwann cells were also shown to intercalate between cancer cells via long protrusions to interrupt cell-cell contacts within the cluster, often causing cancer cell dispersion. Thus, Deborde et al. clearly demonstrate that Schwann cells first make contact with cancer cells, cancer
This direct contact caused entire cancer cells then extend protrusions, and finally Schwann cells intercalate among cancer cells (Figure 1). It is this series of events that most often leads to cancer cell dissociation and migration away from the cluster and toward a Schwann cell (12).

This cancer cell-Schwann cell dynamic appears to contribute to perineural invasion, as Deborde et al. demonstrate that the presence of Schwann cells drastically increases cancer cell invasion into a 3D matrix (12). This invasion is dependent on direct contact between cancer cells and Schwann cells, as neither Schwann cell-released soluble factors nor empty laminin-coated tunnels established by Schwann cells in the matrix were sufficient to promote the same degree of invasion. Thus, Deborde et al. make a strong case that the cellular contact and neuronal guidance functions of Schwann cells specifically contribute to tumor perineural invasion, as paracrine signaling and matrix remodeling have little effect (12).

\section{NCAM1 as a molecular mediator of perineurial invasion}

Deborde et al. provide evidence that immunoglobulin family member neural cell adhesion molecule 1 (NCAM1) may be a key molecular regulator of Schwann cell-cancer cell interactions in perineural invasion (12). Although NCAM1 expression has previously been correlated with perineural invasion in clinical samples $(20,21)$ and implicated in cancer progression and axonal guidance (22-25), a role for NCAM1 in the regulation of perineural invasion has been unexplored. NCAM1 silencing in Schwann cells inhibited their ability to promote cancer cell invasion, protrusions, and recruitment to neurites. In addition, Deborde et al. demonstrate that NCAM1, when overexpressed in cocultured cancer and Schwann cells, localized to the plasma membrane of Schwann cells at sites in which filopodia extended to establish contact with cancer cells. However, overexpression of NCAM1 in NIH 3T3 cells or in NCAM1-silenced Schwann cells was not sufficient to recapitulate the effects of wild-type Schwann cells on promoting cancer cell invasion. In a mouse model of perineural invasion, Deborde and colleagues observed a dramatic decrease in perineural invasion in Ncam1 knockout mice compared with WT animals; however, they 
also observed that sciatic nerve function a clinical indicator of severe perineural invasion - is only partially restored to healthy levels. In clinical pancreatic cancer samples, NCAM1 was expressed by dedifferentiated $\left(\mathrm{GFAP}^{+}\right)$Schwann cells in contact with cancer cells, yet loss of NCAM1 expression in their mouse model of perineural invasion did not seem to affect the dedifferentiation status of Schwann cells in the sciatic nerve (12).

While Deborde et al. make a strong case that NCAM1 plays a key role in promoting Schwann cell-mediated perineural invasion and cancer cell guidance and dissociation, the lack of rescue of NCAM1 overexpression in their in vitro models and the partial therapeutic efficacy of NCAM1 silencing in inhibiting perineural invasion in vivo suggests there are other key regulators of perineural invasion (12). Indeed, other factors, including glial cell-derived neurotrophic factor (GDNF), chemokine receptor CX3CR1, neurotrophin-3, and neurotrophin-4, have been implicated in perineural invasion. However, a thorough and in-depth investigation into the function and mechanism of most of these candidates is still needed $(9,26)$.

\section{Conclusions and future directions}

Although perineural invasion has been clinically described for decades, our mechanistic understanding remains limited. Deborde and colleagues have made exciting contributions toward our understanding of perineural invasion. Schwann cells have been clearly shown to be a novel cell type in the tumor microenvironment that promotes cancer, with a specific role in promoting perineural invasion. By employing detailed live-cell imaging, Deborde and colleagues characterized the precise steps of cancer cell-Schwann cell initial contact, cancer cell protrusion initiation, Schwann cell intercalation, and, ultimately, cancer cell dispersion from cancer cell clusters and toward nerves to promote invasion and cancer spread. Schwann cells seem to repurpose functions involved in normal neuronal guidance and repair to promote tumor spread and progression. This work highlights the importance of direct heterocellular contact between cancer cells and Schwann cells. Additionally, although many molecular players have been impli- cated in the regulation of perineural invasion through correlation and clinical observations, this study demonstrates a specific molecular role for NCAM1 in perineural invasion. Although the data suggest that NCAM1 may not be the single most important regulator of perineural invasion, the results strongly suggest that NCAM1 plays at least a partial role and lay the foundation for future evaluation of other molecular regulators in the functional interactions between Schwann cells and cancer cells. In regards to potential clinical applications, further examination is need to determine whether targeting Schwann cells in general or specific molecular regulators of perineural invasion in particular would be more beneficial for patients. Understanding the drivers and cellular mechanisms of perineural invasion will undoubtedly open potential avenues for therapeutic development and intervention.

\section{Acknowledgments}

S.H. Azam was supported in part by a grant from the National Institute of General Medical Sciences under award 5T32 GM007092.

Address correspondence to: Chad V. Pecot, 450 West Drive, Chapel Hill, North Carolina 27599, USA. Phone: 919.966.4779; E-mail:pecot@email.unc.edu.

1. Lutgendorf SK, et al. Social isolation is associated with elevated tumor norepinephrine in ovarian carcinoma patients. Brain Behav Immun. 2011;25(2):250-255.

2. Hassan S, et al. Behavioral stress accelerates prostate cancer development in mice. JClin Invest. 2013;123(2):874-886.

3. Armaiz-Pena GN, et al. Src activation by $\beta$-adrenoreceptors is a key switch for tumour metastasis. Nat Commun. 2013;4:1403.

4. Shahzad MMKMM. Stress effects on FosBand interleukin-8 (IL8)-driven ovarian cancer growth and metastasis. JBiol Chem. 2010;285(46):35462-35470.

5. Thaker PH, et al. Chronic stress promotes tumor growth and angiogenesis in a mouse model of ovarian carcinoma. Nat Med. 2006;12(8):939-944.

6. Armaiz-Pena GN, Cole SW, Lutgendorf SK, Sood AK. Neuroendocrine influences on cancer progression. Brain Behav Immun. 2013;30(suppl):S19-S25.

7. Magnon C, et al. Autonomic nerve development contributes to prostate cancer progression. Science. 2013;341(6142):1236361.

8. Zhao CM, et al. Denervation suppresses gastric tumorigenesis. Sci Transl Med. 2014;6(250):250ra115.
9. Marchesi F, Piemonti L, Mantovani A, Allavena P. Molecular mechanisms of perineural invasion, a forgotten pathway of dissemination and metastasis. Cytokine Growth Factor Rev. 2010;21(1):77-82.

10. Bapat AA, Hostetter G, Von Hoff DD, Han $\mathrm{H}$. Perineural invasion and associated pain in pancreatic cancer. Nat Rev Cancer. 2011;11(10):695-707.

11. Liebig C, Ayala G, Wilks JA, Berger DH, Albo D. Perineural invasion in cancer: a review of the literature. Cancer. 2009;115(15):3379-3391.

12. Deborde S, et al. Schwann cells induce cancer cell dispersion and invasion. J Clin Invest. 2016;126(4):1538-1554.

13. Jessen KR. Glial cells. Int J Biochem Cell Biol. 2004;36(10):1861-1867.

14. Kaplan S, Odaci E, Unal B, Sahin B, Fornaro M. Chapter 2: Development of the peripheral nerve. Int Rev Neurobiol. 2009;87:9-26.

15. Glenn TD, Talbot WS. Signals regulating myelination in peripheral nerves and the Schwann cell response to injury. Curr Opin Neurobiol. 2013;23(6):1041-1048.

16. Kang H, Tian L, Thompson W. Terminal Schwann cells guide the reinnervation of muscle after nerve injury. J Neurocytol. 2003;32(5-8):975-985.

17. Scheib J, Hoke A. Advances in peripheral nerve regeneration. Nat Rev Neurol. 2013;9(12):668-676.

18. Son YJ, Thompson WJ. Nerve sprouting in muscle is induced and guided by processes extended by Schwann cells. Neuron. 1995;14(1):133-141.

19. Luo XL, Sun MY, Lu CT, Zhou ZH. The role of Schwann cell differentiation in perineural invasion of adenoid cystic and mucoepidermoid carcinoma of the salivary glands. Int J Oral Maxillofac Surg. 2006;35(8):733-739.

20. Chen-Tsai CP, Colome-Grimmer M, Wagner RF Jr. Correlations among neural cell adhesion molecule, nerve growth factor, and its receptors, TrkA, TrkB, TrkC, and p75, in perineural invasion by basal cell and cutaneous squamous cell carcinomas. Dermatol Surg. 2004;30(7):1009-1016.

21. Shang J, Sheng L, Wang K, Shui Y, Wei Q. Expression of neural cell adhesion molecule in salivary adenoid cystic carcinoma and its correlation with perineural invasion. Oncol Rep. 2007;18(6):1413-1416.

22. Campodonico PB, et al. The neural cell adhesion molecule is involved in the metastatic capacity in a murine model of lung cancer. Mol Carcinog. 2010;49(4):386-397.

23. Maness PF, Schachner M. Neural recognition molecules of the immunoglobulin superfamily: signaling transducers of axon guidance and neuronal migration. Nat Neurosci. 2007;10(1):19-26.

24. Saffell JL, Ashton SV, Walsh FS, Doherty P. The changing role of NCAM as a neurite outgrowth-promoting molecule during development. Biochem Soc Trans. 1992;20(2):410-412.

25. Zecchini S, et al. The adhesion molecule NCAM promotes ovarian cancer progression via FGFR signalling. EMBO Mol Med. 2011;3(8):480-494.

26. Roh J, Muelleman T, Tawfik O, Thomas SM. Perineural growth in head and neck squamous cell carcinoma: a review. Oral Oncol. 2015;51(1):16-23. 